\title{
SWEEPING AND STRAINING EFFECTS IN SOUND GENERATION BY HIGH REYNOLDS NUMBER ISOTROPIC TURBULENCE *
}

\author{
Ye Zhou and Robert Rubinstein \\ Institute for Computer Applications in Science and Engineering \\ NASA Langley Research Center \\ Hampton, VA 23681
}

\begin{abstract}
The sound radiated by isotropic turbulence is computed using inertial range scaling expressions for the relevant two time and two point correlations. The result depends on whether the decay of Eulerian time correlations is dominated by large scale sweeping or by local straining: the straining hypothesis leads to an expression for total acoustic power given originally by Proudman, whereas the sweeping hypothesis leads to a more recent result due to Lilley.
\end{abstract}

\footnotetext{
* This research was supported by the National Aeronautics and Space Administration under NASA Contract No. NAS1-19480 while the authors were in residence at the Institute for Computer Applications in Science and Engineering (ICASE), NASA Langley Research Center, Hampton, VA 23681-0001.
} 


\section{Introduction}

The sound radiated by isotropic turbulence was first computed by Proudman ${ }^{1}$ very shortly after the appearance of Lighthill's quadrupole theory ${ }^{2}$ of sound radiation from an arbitrary stochastic velocity field. For the total acoustic power $\bar{p}$ in the far field, Proudman found

$$
\bar{p}=\alpha \rho\left(\frac{u}{c}\right)^{5} \varepsilon
$$

where $c$ is the speed of sound and $\rho$ the density in the far field, $\varepsilon$ is the dissipation rate of the isotropic turbulence, $u$ is defined by

$$
u^{2}=\frac{2}{3} K
$$

where $K$ is the turbulence kinetic energy, and the presumably universal proportionality constant $\alpha$, the Proudman constant, was expressed in terms of the spatial correlation function of the turbulence.

Proudman's calculation was re-examined by Lilley ${ }^{3}$ who noted that Proudman had introduced approximations which suppress dependence on time correlations. This important observation led to a modification of Proudman's formula

$$
\bar{p}=\alpha^{\prime} \rho\left(\frac{\Omega L}{u}\right)^{4}\left(\frac{u}{c}\right)^{5} \varepsilon
$$

where temporal properties appear through the frequency $\Omega$ which is defined so that the time correlation function depends only on the product $\Omega \tau$, where $\tau$ is the time difference. In Eq. (3), $L$ is the integral scale defined by $L=u^{3} / \varepsilon$. By invoking a correction ${ }^{4}$ to the quasinormal hypothesis suggested by Lighthill, Lilley also found that the proportionality constant $\alpha^{\prime}$ depends on the flatness of the single point velocity probability distribution function. However, experimental evidence ${ }^{5}$ indicates that this correction is not large; therefore, this effect will be neglected here.

In evaluating the integrals required by Lighthill's theory, Lilley substituted empirical formulas for the relevant correlation functions, choosing in particular the two time dependence to establish the far field sound spectrum found in numerical simulations by 
Sarkar and Hussaini. ${ }^{6}$ Lilley has argued ${ }^{7}$ that this procedure is reasonable since the temporal covariance depends strongly on the large eddy contribution and therefore should be accessible to direct numerical simulation.

In this letter, Lilley's calculation is repeated using Kolmogorov inertial range forms appropriate to high Reynolds number turbulence. This procedure leads to expressions for acoustic power which contain only universal inertial range quantities. An important feature of Lilley's analysis is the dependence of sound emission on both Eulerian two time

and two point correlations. Whereas there is little doubt that the spatial correlations must follow the Kolmogorov laws $<u(\mathbf{x}+\mathbf{r}) u(\mathbf{x})>\sim r^{2 / 3}$ or equivalently $E(k) \sim k^{-5 / 3}$, there are two plausible alternatives for the time correlations ${ }^{8}$ : temporal decorrelation of the Eulerian velocity field can be caused either by sweeping by the largest energetic scales or by local straining. A definitive discussion of these decorrelation mechanisms and their physical basis is given by Kraichnan ${ }^{9}$; briefly, under the straining hypothesis, temporal decorrelation is a local property of inertial range scales, but under the sweeping hypothesis, it is a nonlocal property of the energy containing range of scales. Although the most recent discussions ${ }^{10,11,12}$ favor the sweeping hypothesis, we examine both cases and find that the local straining hypothesis leads to Proudman's formula Eq. (1), but the sweeping hypothesis leads, after an appropriate substitution for $\Omega$, to Lilley's formula Eq. (3).

\section{Evaluation of the sound radiated by isotropic turbulence}

For time stationary sources, Lilley's approximate form ${ }^{3}$ of Lighthill's theory predicts

$$
\bar{p}=\frac{\rho}{8 \pi^{2} c^{5}} \int d \mathbf{r} U(\mathbf{r})
$$

where the integrand $U$ is given by

$$
\begin{aligned}
U(\mathbf{r}) & =\left.\frac{\partial^{4}}{\partial \tau^{4}}\left[<u_{1}(\mathbf{x}+\mathbf{r}, t+\tau)^{2} u_{1}(\mathbf{x}, t)^{2}>-<u_{1}^{2}>^{2}\right]\right|_{\tau=0} \\
& \left.\equiv \frac{\partial^{4}}{\partial \tau^{4}} \mathcal{U}(\mathbf{r}, \tau)\right|_{\tau=0}
\end{aligned}
$$


where $u_{1}$ is the velocity component in the direction of the line connecting the source and observation point. The quantity $\mathcal{U}$ of Eq. (5) is evaluated in wavenumber space by using the quasi-normal closure in the usual way to obtain

$$
\mathcal{U}(\mathbf{k}, \tau)=\frac{4}{5} \int_{\mathbf{k}=\mathbf{p}+\mathbf{q}} d \mathbf{p} d \mathbf{q} Q(p, \tau) Q(q, \tau)
$$

where $Q(\mathbf{k}, \tau)$ is the correlation function defined by

$$
<u_{i}(\mathbf{k}, t+\tau) u_{j}\left(\mathbf{k}^{\prime}, t\right)>=Q(k, \tau) P_{i j}(\mathbf{k}) \delta\left(\mathbf{k}+\mathbf{k}^{\prime}\right)
$$

and

$$
P_{i j}(\mathbf{k})=\delta_{i j}-k^{-2} k_{i} k_{j}
$$

Following Lilley, we set $\mathbf{k} \rightarrow 0$ in Eq. (6), corresponding to the far field approximation. Then

$$
\bar{p}=\frac{(2 \pi)^{3} \rho}{8 \pi^{2} c^{5}} \frac{\partial^{4}}{\partial \tau^{4}} \frac{4}{5} \int 4 \pi p^{2} d p Q(p, \tau)^{2}
$$

Now write the two time correlation as

$$
Q(p, \tau)=Q(p) r(p, \tau)
$$

where

$$
4 \pi p^{2} Q(p)=E(p)
$$

is the energy spectrum and $r$ has the similarity form

$$
r(p, \tau)=r(\tau \theta(p))
$$

with

$$
\theta(p)= \begin{cases}V p & \text { sweep } \\ \varepsilon^{1 / 3} p^{2 / 3} & \text { strain }\end{cases}
$$

Whereas the decorrelation time $\theta^{-1}$ is a local inertial range property under the straining hypothesis, the sweeping hypothesis makes the decorrelation depend on the sweeping velocity $V$, which is not an inertial range property, but an entirely independent property of the energy containing range. 
Substituting Eqs. (8)-(11) in Eq. (7) leads to

$$
\bar{p}=\left.\frac{1}{5} \frac{\rho}{c^{5}} \frac{\partial^{4}}{\partial \tau^{4}} \int_{0}^{\infty} d p p^{-2} E(p)^{2} r(\tau \theta(p))^{2} \quad\right|_{\tau=0}
$$

Differentiating Eq. (12) under the integral sign,

$$
\bar{p}=\frac{1}{5} \frac{\rho}{c^{5}} \int_{0}^{\infty} d p p^{-2} E(p)^{2} \mathcal{R} \begin{cases}V^{4} p^{4} & \text { sweep } \\ \varepsilon^{4 / 3} p^{8 / 3} & \text { strain }\end{cases}
$$

where

$$
\mathcal{R}=\left.\frac{d^{4}}{d \xi^{4}} r(\xi)\right|_{\xi=0}=2 r^{(4)}(0) r(0)+6\left[r^{(2)}(0)\right]^{2}
$$

is a universal inertial range constant.

The integrals in Eq. (13) will be evaluated assuming a truncated Kolmogorov inertial range

$$
E(p)= \begin{cases}C_{K} \varepsilon^{2 / 3} p^{-5 / 3} & \text { for } p \geq k_{0} \\ 0 & \text { for } p \leq k_{0}\end{cases}
$$

Both integrals exhibit power law divergence in the limit $k_{0} \rightarrow 0$ : the integral for the sweeping hypothesis diverges as $k_{0}^{-1 / 3}$ and the integral for the straining hypothesis diverges as $k_{0}^{-5 / 3}$. This divergence indicates as expected that sound generation is dominated by contributions from the most energetic scales. Defining the inertial range energy

$$
K=\int_{k_{0}}^{\infty} E(k) d k=\frac{3}{2} C_{K} \varepsilon^{2 / 3} k_{0}^{-2 / 3}
$$

where $C_{K}$ is the Kolmogorov constant, we note that in the infinite Reynolds number limit in which $k_{0} \rightarrow 0$,

$$
\bar{p} \sim \begin{cases}K^{1 / 2} & \text { sweep } \\ K^{5 / 2} & \text { strain }\end{cases}
$$

Using these results, evaluation of the integrals in Eq. (9) leads to

$$
\bar{p}=\varepsilon\left(\frac{u}{c}\right)^{5} \begin{cases}\alpha_{\text {sweep }}(V / u)^{4} & \text { sweep } \\ \alpha_{\text {strain }} & \text { strain }\end{cases}
$$

For the proportionality constants, we obtain

$$
\begin{aligned}
\alpha_{\text {sweep }} & =\frac{3}{5} \mathcal{R} C_{K}^{3 / 2} \\
\alpha_{\text {strain }} & =\frac{9}{25} \mathcal{R} C_{K}^{-1 / 2}
\end{aligned}
$$


The inertial range constant $\mathcal{R}$ may be different for the straining and sweeping hypotheses, but this fact has not been made explicit to simplify the notation.

The acoustic power given by Eq. (15) for strain evidently coincides with Proudman's result Eq. (1). Lilley's formula Eq. (2) also reduces to Proudman's if the characteristic frequency $\Omega$ is an inertial range property, for example if $\Omega \sim \varepsilon / K$. But if $\Omega$ is chosen to be a sweeping frequency, say $\Omega \sim V / L$, then Lilley's formula coincides instead with Eq. (15) for sweep.

\section{Evaluation of the Proudman constant}

To evaluate the proportionality constant $\alpha_{\text {strain }}$, we use Kaneda's ${ }^{13}$ result

$$
r(\xi)=\exp \left(-0.81 \pi \xi^{2} / 4\right)
$$

derived for a Lagrangian modification of the direct interaction approximation in which time correlations are dominated by local strain. In this case, $\mathcal{R}=12.7$ and therefore

$$
\alpha_{\text {strain }}=3.6
$$

To evaluate the proportionality constant $\alpha_{\text {sweep }}$, we use Kraichnan's result ${ }^{14}$ that the time correlation function for the sweeping hypothesis is

$$
r(\xi)=\frac{J_{1}(2 \xi)}{\xi}
$$

therefore $\mathcal{R}=10$. Substituting in Eq. (16),

$$
\alpha_{\text {sweep }}=6 C_{K}^{3 / 2}=12.0
$$

Woodruff has noted ${ }^{15}$ that since the time correlation functions of Eqs. (17) and (18) only enter Eq. (13) through their derivatives at zero, formulas for the constant $\mathcal{R}$ could be obtained from Taylor series solutions of the Navier Stokes equations. By expressing these derivatives in terms of single time moments without invoking quasi-normality, a generalization of the Lighthill ${ }^{4}$ relation could be derived. 
To assess the agreement between the present theory and Lilley's, it is necessary to compare values of $\alpha^{\prime}(\Omega L / u)^{4}$ and $\alpha_{\text {sweep }}(V / u)^{4}$. Table I contains representative values of the first quantity; the Strouhal number is the factor $S t=\Omega L / u$ of Lilley's theory. $S t$ is probably $^{7}$ somewhat larger than 1.0 . The value $S t=1.25$ which appears in Table I is an upper bound, not an experimental or theoretical value. Refs. 3 and 5 attempt to extract the parameters of Lilley's theory from isotropic turbulence data. In Ref. 6, the sound radiated by isotropic turbulence was determined by numerical simulation; however, recent reconsideration ${ }^{16}$ of this calculation suggests that the result may not be entirely reliable. With $S t$ in the range $1.00-1.25, \alpha^{\prime} S t^{4}$ takes values between 0.7 and 8.8 .

Like the Strouhal number, the ratio $V / u$ is essentially nonuniversal since it depends on properties of the energy containing range. Table II contains data taken from a recent survey $^{17}$ of experiments with well defined inertial and energy containing ranges. The experiments can be identified from Ref. 17 through the value of $R e_{\lambda}$. The sweeping velocity $V$ is evaluated from the energy in the scales below the $5 / 3$ range. The values of $12(\mathrm{~V} / \mathrm{u})^{4}$ in Table II are generally within the range found in Table I. However, the data of Table I matches the entire energy spectrum including the energy containing range, whereas the present theory ignores the contribution of these scales to sound radiation. This suggests that the theoretically computed value of $\alpha_{\text {sweep }}$ in Eq. (19) might be somewhat too large. ${ }^{7}$ In applying experimental results ${ }^{5,17}$ to an isotropic theory, we assume that the overall energetics of possibly inhomogeneous and anisotropic turbulence does not differ greatly from ideal isotropic turbulence.

In both the present theory and Lilley's, the fourth power dependence on the external parameters $\Omega$ and $V$ is striking. In particular, the present theory predicts that decorrelation by the energy containing range strongly enhances the radiation of sound; as noted previously, the sound radiated by the energy containing scales themselves has not been included in the present calculation. 


\section{The acoustic power spectrum}

Taking the Fourier transform of Eq. (12) leads to the formula for the acoustic power spectrum

$$
\left.\bar{p}(\omega) \sim \frac{\rho}{c^{5}} \int_{0}^{\infty} d p p^{-2} E(p)^{2} r(\zeta) * r(\zeta)\right|_{\zeta=\omega / \theta} \theta^{-1}
$$

where $r(\zeta)=r(\omega / \theta(p))$ is the Fourier transform of the time correlation function defined by Eq. (10) and * denotes convolution with respect to the variable $\zeta$. By introducing the change of variables $q=\omega / \theta(p)$ in Eq. (20), we find

$$
\bar{p}(\omega) \sim \frac{\rho \varepsilon}{c^{5}} \begin{cases}\varepsilon^{1 / 3} V^{13 / 3} \omega^{-4 / 3} & \text { sweep } \\ \varepsilon^{5 / 2} \omega^{-7 / 2} & \text { strain }\end{cases}
$$

The considerable difference in the scaling exponents might make an experimental test of the sweeping and straining hypotheses possible. The spectral scaling for sweep in Eq. (21) can be compared to the $\omega^{-2}$ scaling ${ }^{3}$ which has been found to give a good description of jet mixing noise. ${ }^{18}$ It should be noted that Proudman's theory ${ }^{1}$ does not provide an expression for the acoustic power spectrum.

\section{Conclusions}

The calculation of sound radiation by isotropic turbulence has been considered from the viewpoint of analytical theories of turbulence. The calculation is accordingly based on universal inertial range descriptors of turbulence; empirical space and time correlation functions are not used. The Proudman constant is expressed theoretically in terms of the Kolmogorov constant, a time correlation constant, and various purely geometric factors. The calculation shows a significant effect of the behavior of Eulerian time correlations on sound radiation. If decay of time correlations is dominated by local straining, then sound radiation is given by Proudman's original formula ${ }^{1}$ and the acoustic power spectrum follows an $\omega^{-7 / 2}$ law; whereas the more plausible hypothesis that decay of time correlations is dominated by sweeping by the largest energetic scales results in a formula much closer to Lilley's ${ }^{3}$, and the acoustic power spectrum follows an $\omega^{-4 / 3}$ law. 


\section{TABLE I}

$\begin{array}{lccc} & \alpha^{\prime} & S t & \alpha^{\prime} S t^{4} \\ \text { Ref. 3 } & 3.2-3.6 & 1.25 & 7.8-8.8 \\ \text { Ref. 5 } & 0.7-2.7 & 1.25 & 1.7-6.5 \\ \text { Ref. 6 } & & & 2.6\end{array}$

\section{TABLE II}

$\begin{array}{ccc}R e_{\lambda} & V / u & 12(V / u)^{4} \\ 3180 & .48 & 0.6 \\ 1450 & .86 & 6.4 \\ 850 & .65 & 2.2 \\ 540 & .59 & 1.5 \\ 308 & .84 & 5.9\end{array}$

\section{Acknowledgments}

We would like to acknowledge an extensive and beneficial correspondence with G. M. Lilley, and to thank M. Y. Hussaini, R. H. Kraichnan, and S. Sarkar for helpful comments

\section{References}

1. I. Proudman, "The generation of noise by isotropic turbulence," Proc. Roy. Soc. London, A214, 119 (1952).

2. M. J. Lighthill, "On sound generated aerodynamically: 1. General theory," Proc. Roy. Soc. A211, 1107 (1952).

3. G. M. Lilley, "The radiated noise from isotropic turbulence revisited," Theor. Comput. Fluid Dyn. 6, 281 (1994).

4. M. J. Lighthill, "An estimate of the covariance of $T_{x x}$ without using statistical assumptions," in Computational Aeroacoustics J. C. Hardin and M. Y. Hussaini, eds., Springer-Verlag (1993).

5. Y. Zhou, A. A. Praskovsky and S. Oncley, "On the Lighthill relationship and sound generation from isotropic turbulence," Theor. Comput. Fluid Dyn. 7, 355 (1995).

6. S. Sarkar and M. Y. Hussaini, "Computation of the sound generated by isotropic turbulence," ICASE Report 93-74 (1993). 
7. G. M. Lilley, private communication.

8. H. Tennekes, "Eulerian and Lagrangian time microscales in isotropic turbulence,"J. Fluid Mech. 67, 561 (1975).

9. R. H. Kraichnan, "Kolmogorov's hypotheses and Eulerian turbulence theory," Phys. Fluids 7, 1723 (1964).

10. S. Y. Chen and R.H. Kraichnan, "Sweeping decorrelation in isotropic turbulence," Phys. Fluids A1, 2019 (1989)

11. M. Nelkin and R. Tabor, "Time correlations and random sweeping in isotropic turbulence," Phys. Fluids A 2, 81 (1990).

12. A. Praskovsky, E. Gledzer, M. Karyakin and Ye Zhou, "Sweeping decorrelation hypothesis and energy-inertial scale interaction in high Reynolds number flows," J. Fluid Mech. 248, 493 (1993).

13. Y. Kaneda, "Renormalized expansions in the theory of turbulence with the use of the Lagrangian position function," J. Fluid Mech. 107, 131 (1981).

14. R. H. Kraichnan, "The structure of turbulence at very high Reynolds number," J. Fluid Mech. 5, 497 (1959).

15. S. L. Woodruff, private communication.

16. J. Whitmire and S. Sarkar, "The computation of flow generated sound using an acoustic analogy," CEAS/AIAA paper 95-038 (1995).

17. S. G. Saddoughi and S. V. Veeravalli, "Local isotropy in turbulent boundary layer at high Reynolds number," J. Fluid Mech. 268, 333 (1994).

18. G. M. Lilley, "Jet noise: classical theory and experiment," in Aeroacoustics of flight vehicles: theory and practice, H. H. Hubbard (ed.), NASA Reference Publication 1258 vol 1 (1991). 